Research Article

\title{
Application of Multislice Spiral CT Volume Reconstruction in Congenital Microtia Reconstruction
}

\author{
Hongjuan Jin (iD) and Weihai Peng (D) \\ Department of Plastic and Reconstructive Surgery, The First Hospital of Jilin University, Changchun, Jilin 130021, China \\ Correspondence should be addressed to Weihai Peng; pengwh@jlu.edu.cn
}

Received 20 June 2021; Accepted 8 September 2021; Published 7 October 2021

Academic Editor: Gustavo Ramirez

Copyright (c) 2021 Hongjuan Jin and Weihai Peng. This is an open access article distributed under the Creative Commons Attribution License, which permits unrestricted use, distribution, and reproduction in any medium, provided the original work is properly cited.

Objective. To investigate the application of multislice spiral CT in volume reconstruction of congenital microtia. Methods. Sixty patients who underwent auricle reconstruction in Otolaryngology Hospital of our hospital from April 2020 to April 2021 were selected. All patients had no mental disorders and normal cognitive ability and volunteered to participate in this study. Multislice spiral CT was used to evaluate the diagnostic accuracy of multislice spiral CT by scanning the morphology of the ossicular chain and the bone destruction of the selected patients. Results. MSCT can clearly display the structure of ear. Conclusion. MSCT can clearly reflect the external ear and the structure of the ear in patients with congenital microtia and distinguish the different types of patients obviously. Multiplane reconstruction and volume reconstruction can clearly display the fine structure of the patient's ear, which has important reference value for surgery.

\section{Introduction}

Congenital microtia, also known as microtia, is characterized by symptoms such as severe underdevelopment of auricle, atresia of external auditory canal, and impairment of hearing ability. In some patients, congenital microtia and atresia of external auditory canal will appear simultaneously, and the patient's middle ear and face are not fully developed. Congenital microtia is a kind of congenital malformation with unknown causes, which is characterized by congenital auricle dysplasia, often accompanied by external auditory canal atresia, middle ear, and maxillofacial malformations. At present, the most common treatment methods are surgical reconstruction of the auricle and hearing reconstruction. Currently, clinical and CT studies on various diseases and normal anatomical structures of the ear are gradually increasing. High-resolution CT (HRCT) and multislice spiral CT (MSCT) three-dimensional reconstruction techniques such as surface shadow are used for normal structures and diseases such as ossicular chain and inner auricular labyrinth maximum/minimum intensity projection (MIP/MINIP), maximum/minimum intensity projection (SSD), multiplanar reconstruction (MPR), virtual endoscopy (VE), and other studies have been reported in recent years. In the past, volume rendering (VR) and transparent reconstruction (Ray Sum) techniques have been widely used in the studies on the relationship between abdominal organs, blood vessels, and tumors, but there are few reports on the ear, especially the inner ear, which are still in the preliminary exploration stage, and the study on the ossicle has not been reported yet. As VR is a three-dimensional reconstruction technology in the true sense of a higher form, it has the unique advantages of $100 \%$ utilization of the scanned volume of content volume data. Compared with MIP and SSD, which only use $10 \%$ of volume data after editing, the images obtained are more realistic, three-dimensional, and clear. The purpose of this study is to further study the application value of VR in displaying the anatomical structures of normal middle ear and inner ear, as well as the surface and auditory nerve in the internal auditory canal, and to compare VR with other postprocessing techniques to evaluate the superiority of VR.

At present, there are mainly two kinds of surgical methods for ear reconstruction: one is a variety of nondilatation methods based on Tanzer method; the other is the soft tissue 
dilation of the skin behind the ear. The scaffold materials were autogenous costal cartilage and artificial materials. Autogenous costal cartilage is recognized as the best scaffold material because of its good histocompatibility and sufficient amount. The selection of ear reconstruction methods should be combined with the specific classification of patients' ear deformity and the physiological conditions of local soft tissues. For patients with abundant residual ear tissue and loose and thin skin behind ear, the use of nonexpansion method can achieve good stentcovered skin flap while avoiding dilator-related complications and shorten the surgical cycle of patients. For patients with thick local skin and poor laxity, the ear stent can be covered with sufficient flap by dilatation method, and the details of the ear stent can be better displayed. In order to better develop personalized ear stents for patients, digital technology was used for preoperative evaluation of patients with costal cartilage and ear deformities, intraoperative guidance of ear stent expansion, and postoperative evaluation of reconstructed ear morphology. Many practices have proved that digital technology can reduce the amount of costal cartilage cutting, reduce the unnecessary operation time during the operation, and achieve a good surgical effect. Digital technology can also be used to train young doctors. Although the existing techniques have achieved good surgical effects, there are still some problems, such as the use of autogenous costal cartilage in the reconstructed ear, which is not as soft as the natural ear, the difficulty of costal cartilage harvesting and carving due to calcification, the instability of the ear scaffold, the related complications caused by dilators, and there is still no good treatment method for complex and difficult cases. To solve these problems, we hope that tissue engineering and 3D bioprinting technology may revolutionize the treatment of microtia. In recent years, a major breakthrough has been made in the synthesis of tissue-engineered ear cartilage scaffolds. Cao Yilin et al. combined it with 3D printing technology and applied tissue engineered ear cartilage to 5 children with small ear reconstruction, with the longest follow-up of 2.5 years, and achieved satisfactory aesthetic effects. However, the technique is still in clinical trial stage, and its large-scale clinical application still needs unremitting exploration.

\section{General Data and Methods}

2.1. General Information. Sixty cases (60 ears) of congenital microtia admitted to our hospital from April 2020 to April 20121 were selected as the subjects for clinical analysis. Retrospective analysis was mainly performed, with 30 patients receiving retroauricular flap combined with autogenous costal cartilage transplantation, and 30 patients receiving dilatation combined with autogenous costal cartilage transplantation. There were 33 male patients, ranging in age from 6 to 24 years old, with an average age of $15.57 \pm 0.42$ years, and 27 female patients. Their age ranged from 7 to 23 years, with an average age of $15.21 \pm 0.37$ years.

2.2. Inclusion Criteria. (1) All patients had congenital microtia. (2) All patients had unilateral ear deformity. (3) All patients ranged in age from 6 to 24 years old. (4) All patients and their families are aware of and approved this study. (5) This study has been approved by the ethics committee of the hospital.

2.3. Exclusion Criteria. (1) Patients with severe coagulation dysfunction. (2) Patients with severe cardiovascular and cerebrovascular diseases and liver and kidney diseases. (3) Patients with severe blood diseases. (4) patients with mental disorders, behavioral disorders, and language disorders. (5) Patients who did not meet the above inclusion criteria. The basic data of the two groups were comparable $(P>0.05)$.

\section{Research Methods}

3.1. Inspection Method. The examiner was in a supine position, using a Siemens Somatam Definition CT scanner. The scanning range ranged from the upper edge of the rock bone to the lower edge of the external auditory canal. Scanning parameters are set as follows: tube voltage, $120 \mathrm{kV}$; effective tube current, $220 \mathrm{mAs}$; collimator, $64 \times 0.6 \mathrm{~mm}$; layer thickness, $0.6 \mathrm{~mm}$; and matrix, $512 \times 512 \mathrm{~mm}$. Image processing method: after the scanning was completed, the data were transmitted to MMWP workstation to process the scanning results, and the structure of the examined middle ear was reconstructed by using multiplane reconstruction technology and volume reconstruction technology. Each section of the multiplane reconstruction image was obtained by adjusting the observation angle. The observation window level was set as $800 \mathrm{HU}$, and the width of the observation window was set as $4000 \mathrm{HU}$ [1]. By using volume reconstruction technology, the anatomical structure of the inner ear, tympanum, and ossicular chain can be observed in three dimensions by changing the range of thresholds, and the three-dimensional morphology of the ossicular chain can be reconstructed, so as to observe the morphology of the ossicular chain and the degree of bone destruction of the ear bone from different angles. The threshold range is generally set as $-490 \sim 700$. In addition, the labyrinth, semicircular canal, and cochlea of the inner ear were remodeled by volume reconstruction technology, and the above structures were simultaneously displayed in the temporal bone by combining transparency technology and image fusion technology, so as to observe the whole lesion more comprehensively and intuitively. Image analysis [2]: the image quality was evaluated by two or more competent technologists. In the reconstruction images, the judgment of the damage of the ossicles and the location of the damage was evaluated by two or more radiologists. If the two doctors' evaluation results were different, another physician was asked to evaluate the image quality.

3.2. Postprocessing Technology. The image postprocessing workstation is MXView3.5 version. The ultrahigh filtering algorithm (Filter D) is used to carry out intensive reconstruction of the original image with a reconstruction interval of $0.1 \mathrm{~mm}$ and a matrix of $1.024 \times 1.024$ to obtain $300-400$ images. On this basis, conventional VR, 2D MPR, SSD, CTVE, MIP/MINIP, and other $3 \mathrm{D}$ reconstruction were performed in each of the subjects, mainly to observe the ossicle of the middle ear, the 
labyrinth of the inner ear membrane, and the face and auditory nerve in the inner auditory canal. VR: the thin layer slider SLAB was used to thin slice it from different directions of transverse, coronal, and sagittal and 12 images could be obtained from each direction. To obtain a single structure of ossicle or membranous labyrinth, all the surrounding structures that are not needed in each layer of images after thin-cut are cut off, and then all the retained structures are fused by default. Double threshold technique was used to display membranous labyrinth. First, the threshold value of decay bone structure and surrounding structure to disappear was about $\pm 200 \sim(1100 \pm 100)$, and then the threshold value of membranous labyrinth was about $(-800 \pm 100) \sim \pm 200$. Display ossicle thresholds: upper limit: 747 926; lower limit: 259 727. VE: find relate in the window of voyage, rotate to find the left and right sides, click corresponding to the external ear hole, move the layer, and press the mouse to move to find the auditory bone and the part of interest. The image can be enlarged by rotation. The upper limit of threshold is 500 1000, and the lower limit is $-60 \sim-300$.

3.3. Statistical Analysis. The image data of the selected cases in this group were statistically processed by statistical software (SPSS15.0 version). The chi-square test was used to compare the counting data, and the difference method was used to test the measurement data, which was called $t$-test $(P<0.05$ was considered to be statistically significant).

3.4. Bears Fruit. The normal structural capacity of the ear is shown in Figures 1-12. It can be seen from Figures 1 and 12 that VR can achieve the best display of the fine structures of the ear, followed by MPR and SSD, which can clearly display the small ossicle of the middle ear and the labyrinth of the inner ear, while the elliptic sac, balloon, vestibular nerve, cochlear nerve, cochlear window, vestibular window, and other structures are not displayed or poorly displayed. The display ability of MIP and MINIP is similar to SSD, but the display ability of auditory bone and inner ear is not as clear as SSD. Ray Sum showed a good resolution only for the labyrinth and the internal auditory canal, but could not show the resolution for other middle ear and facial nerve structures. VE was only good in the ossicle and middle ear, but poor in the inner ear. Evaluation criteria: each method was observed by 2 doctors, and if the opinions were different, the third doctor was asked to discuss and unanimously approve the method. Comparison of several reconstruction methods:VR, MIP/MINIP, SSD, and MPR could simultaneously display the ossicle of the middle ear and the labyrinth of the inner ear, but VR showed most clearly (Figure 1-9). The labyrinths of the inner eardrum were better displayed by VR and Ray Sum (Figures 3-7, 10). The ossicle structure of the middle ear was clearly displayed by VR and VE (Figures 1, 2, 12).

In Figures 1 and 2, the transverse and coronal images obtained by VR thin-cut technique can show the fine structures of the ossicle completely and clearly and intuitively. The long process of the incus is parallel to the malleus stem, the stirrup joint is in the shape of " $L$," and the semicircular canal and the labyrinth of the cochlear bone can be seen (arrow).

In Figure 3, the three-dimensional structure of the labyrinth can be clearly displayed, and the elliptic sac, balloon, facial nerve, and vestibular cochlear nerve can be seen (basically the MRI effect can be achieved (arrow)). In Figure 4, the cochlear nerve entering the labyrinth of the cochlear membrane as well as the direction and labyrinth of the vestibular nerve (arrow) can be observed.

Figure 5 clearly shows membrane-labyrinthine stereoscopic structure (arrow). Figure 6 clearly shows the threedimensional structures and relationships of the cochlea and the three semicircular canals, with few artifacts, which were comparable to MRI.

Figure 7 shows the right side of the display surface acoustic nerve, vestibular, and part of the cochlea, semicircular canal, left for the three-dimensional structure of three semicircular canal (arrow). In Figure 8, thin slice images can be observed within the hear of meal, auditory nerve, and the first and second period of the three-dimensional structure of facial (arrow).

Figure 9 clearly displays the bone (membrane) labyrinth (arrow). Figure 10 clearly displays the cochlear membrane labyrinth (arrow) in stereo.

In Figure 11, only part of the auditory bone structure can be stereoscopic display, and there are many trap-like artifacts. Figure 12 displays each structure and image of auditory bone.

\section{Discussion}

4.1. MSCT Technique Discussion. MSCT combines multilayer scanning technology of second level and sub-millimeter level and a variety of powerful post-processing functions, so that it is superior to single-layer spiral CT in scanning the microstructure of inner ear and the qualitative and quantitative of tumor, because its scanning speed is $10 \sim$ 100 times that of single-layer spiral CT, and can complete the scanning of wider and thinner scanning layers at the same time. The application of various posttreatment techniques in clinic was discussed. Although conventional CT, single-slice spiral CT, and HRCT can display the structure and lesions of the middle and inner ear, they are mainly two-dimensional images and lack integrity and continuity. Because of small and complex structure of the ear, compared with other parts of the body, its postprocessing reconstruction need to use very thin, with very high resolution of the original image, while continuous scanning is also required. MSCT meets this demand with its advantages of fast, ultra-thin, and no continuous scanning interval, which not only reduces the impact of some volume effects, moreover, the spatial resolution, cross-sectional signal-to-noise ratio, and contrast of the image are improved. Through various post-processing and reconstruction, the microcomplex stereo images of the middle ear and inner ear can be obtained. The normal anatomy and various lesions can be observed very intuitively and deeply, so as to make a clear diagnosis, which will greatly help the surgeon to formulate a correct operation plan, teaching, and scientific research. 


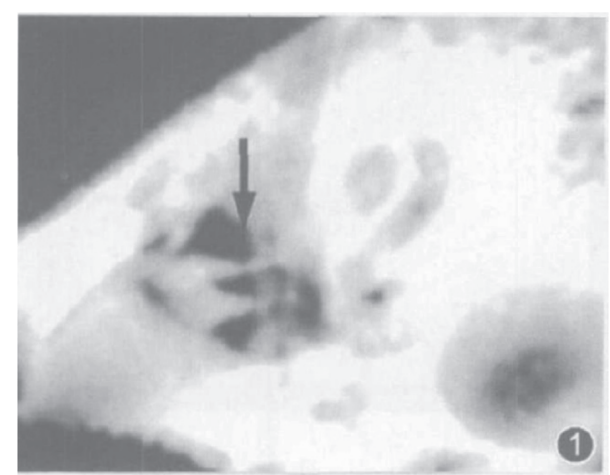

FIGURE 1: The transverse images.

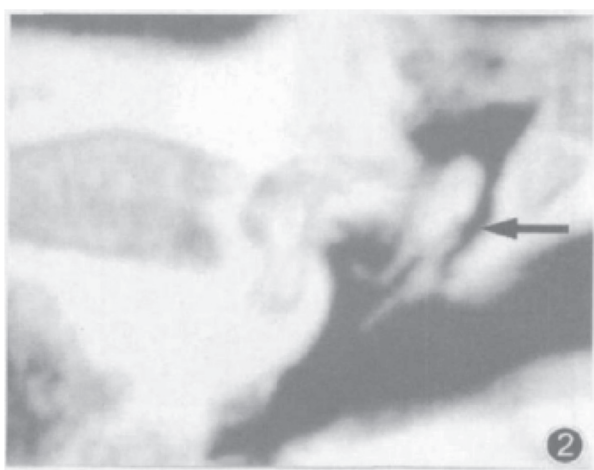

Figure 2: The coronal images.

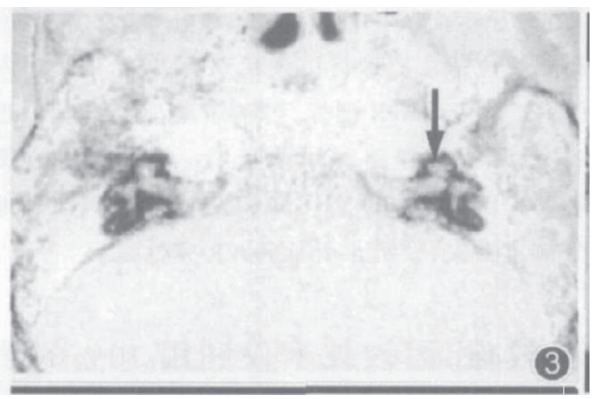

FIGURE 3: VR transverse images of the labyrinth.

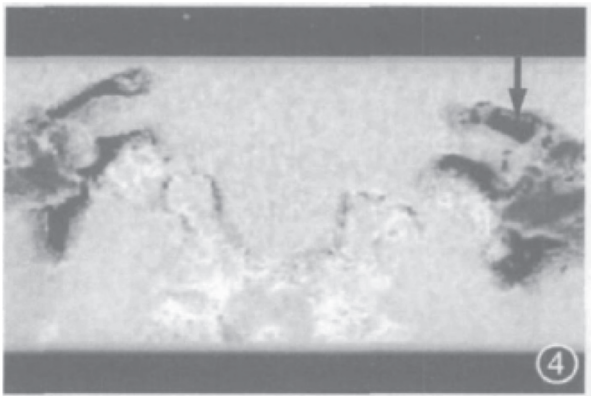

FIGURE 4: 4VR coronal thin-section images.

4.2. Comparison of Multiple Postprocessing Techniques for Multislice Spiral CT. Postprocessing techniques of MSCT mainly include maximum density projection (MIP), surface

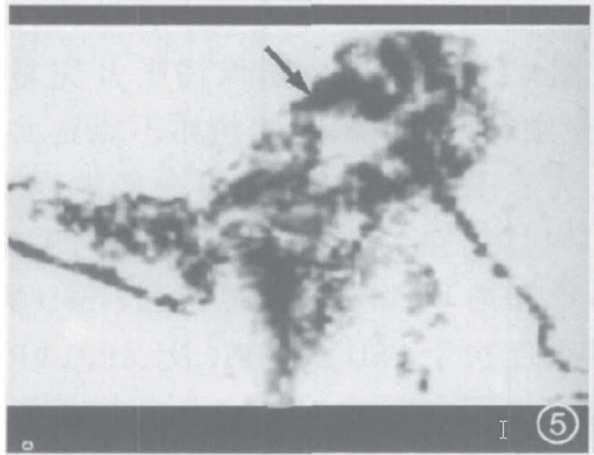

FIGURE 5: VR sagittal thin-cut image.

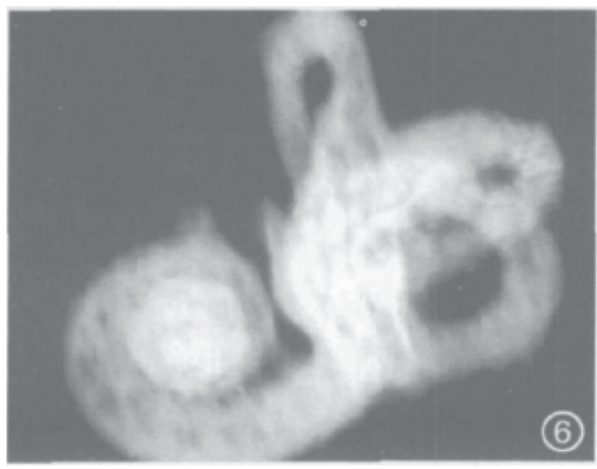

FIGURE 6: VR images of the membranous labyrinth.

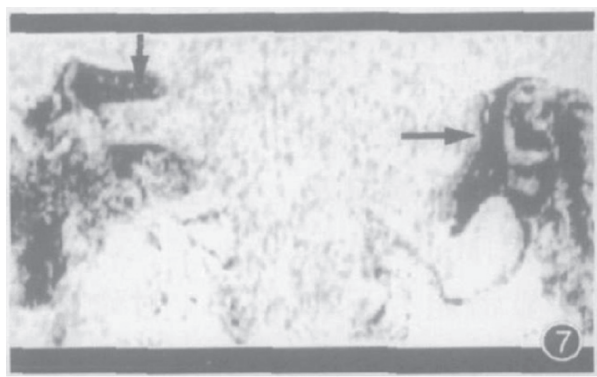

FIGURE 7: VR coronary (asymmetric) binaural put thin slice images.

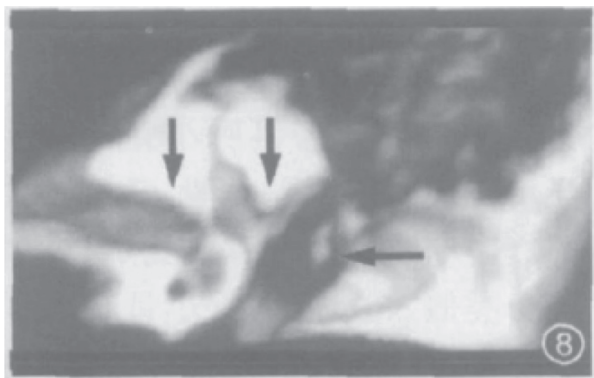

FIGURE 8: VR coronary ear bone.

occlusion (SSD), volume reproduction (VR), simulated endoscope (VE), multiplane reconstruction (MPR), surface reconstruction (CPR), and 3D cutting and fusion. Based on 


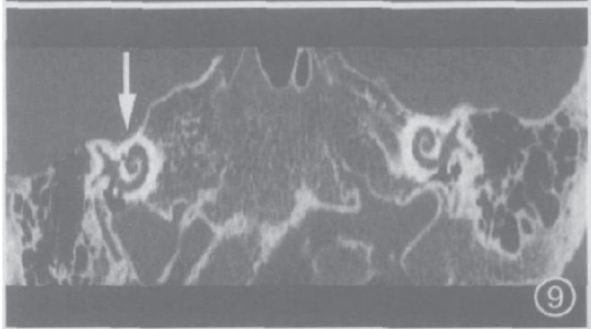

FIGURE 9: MPR image.

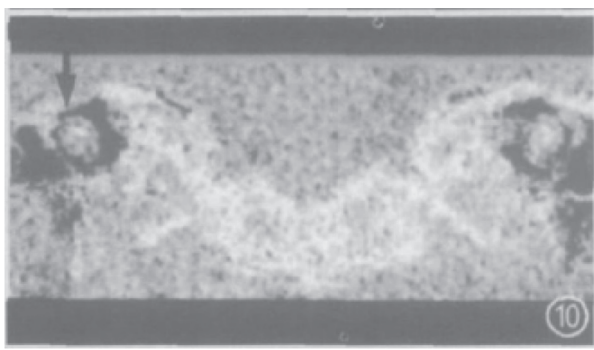

Figure 10: Ray SUM image.

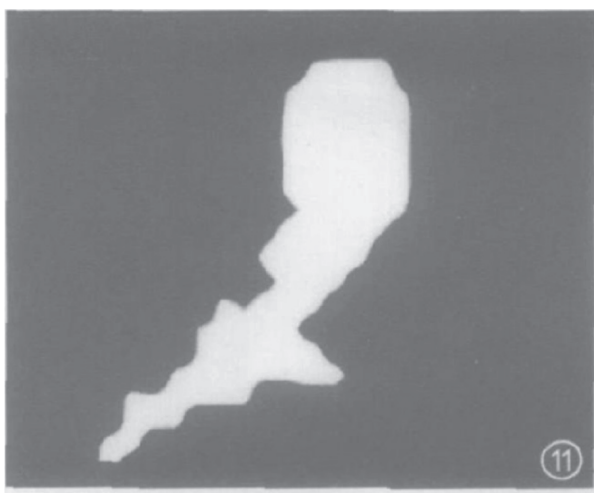

FIGURE 11: SSD image of auditory bone.

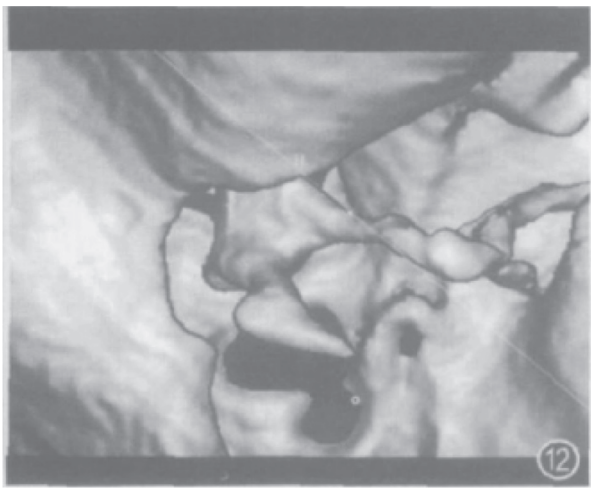

FIgURE 12: CTVE image of auditory bone.

many domestic studies, the posttreatment techniques and methods of congenital microtia are mainly applied in MIP and VR technology and optimized with 3D cutting and fusion tools. The maximum density projection method (MIP) is to carry out projection imaging on the maximum intensity CT value of each pixel in the volume tissue or physical object through which the projected diameter line passes, mainly to show the density difference between the tissues, so the image contrast is very high. The success or failure of SSD technique is closely related to the adjustment of CT threshold. SSD and MIP techniques were not effective in displaying mild auricular stenosis. Volume reproduction (VR) technology is based on all the CT data of the device volume scanning, which is more complete than the volume information of the surface occulting method. It is the most commonly used postprocessing technology in our country at present and can provide clinical medical image information accurately and intuitively. In conclusion, MIP, SSD, VR, and other postprocessing techniques have their own characteristics. The maximum density projection (MIP) can show more distal microtia and its fine structure distribution, but its disadvantage is that the microtia has too much overlapping bone structure. VR technology combines the advantages of the above multiple postprocessing technologies. It not only has a good sense of spatial stereo in the reconstructed image but also can show the microtia situation as a whole and can show the origin location of microtia and its relationship with surrounding structures. The fusion technology of multi-slice spiral CT and VR is based on the automatic target selection (aos-vr) processing technology of postprocessing workstation. The image threshold and color matching are reasonably adjusted mainly through VR postprocessing tools such as adding, deleting, and cutting. VR orthotopic images of microauricular and microauricular malformation were established in each reconstructed image frame, and they were fused into one image. Requirements of VR fusion image technology during postprocessing reconstruction: (1) pay attention to maintaining the integrity of the image during adding or removing structure operation. (2) Necessary and reasonable CT threshold adjustment should be carried out. (3) Choose the appropriate color collocation, need eye-catching, intuitive, easy to distinguish as the principle. Compared with other processing and reconstruction technologies, VR image fusion technology has the following advantages: (1) extract a tissue structure or organ separately for reconstruction, adjust its threshold value and set the color degree without affecting other structures, and the three-dimensional image will be clear and complete after fusion. (2) The three-dimensional fusion image is spatially fused without changing the opacity and removing the interference from the irrelevant junction image structure. (3) The spatial adjacent relationship between the VR postprocessing fusion images is clear and intuitive and can be observed continuously from all levels of rotation. AoS-VR fusion technology should be mainly adopted in the postprocessing of ears, and other maximum density projection and multiplane reconstruction technologies should be used as auxiliary means. VR images of malformed small ears and normal ears can be reconstructed by adjusting various image parameters (opacity, color, etc.) through a variety of postprocessing tools and then fused into an image through image processing. Thus, the structure of 
the ear can be observed one by one, and the shape, size, position, direction, and the relationship between each other can be diagnosed clearly. Therefore, VR fusion technology should be preferred in the posttreatment and reconstruction technology of microtia reconstruction surgery, and a comprehensive analysis of microtia should be carried out to provide help for the selection of preoperative treatment.

\section{Summary}

The MDCT volume reconstruction of microtia and reformation of patients with preoperative and postoperative observation of the small ear structure is more apparent, can effectively improve the success rate of the small ear deformity reconstructive surgery, and reduce the risk of microtia reconstruction surgery and fitment and microtia for surgical patients with preoperative and postoperative accurate diagnostic information. However, there are still many deficiencies in this study. The most important aspect is the small sample size due to the complexity of auricle reconstruction surgery and the limited annual operation volume in hospitals [3-7].

\section{Data Availability}

The data used to support the findings of this study are available from the corresponding author upon request.

\section{Conflicts of Interest}

The authors declare no conflicts of interest.

\section{References}

[1] L. Zhang, C. Ji, and Y. Wang, "Postoperative nursing care of total auricle reconstruction after hearing reconstruction for congenital microtia," Chinese Journal of Practical Nursing, vol. 26, no. 4B, pp. 44-45, 2010.

[2] X. Liu, B. Pan, and C. Wang, "Application of personalized earmuff in auricle reconstruction with dilator for microauricular malformation," Chinese Journal of Nursing, vol. 44, no. 11, p. 1044, 2009.

[3] Y. Wang, L. He, L. Li, C. Weiwei, J. Haiyue, and S. Qiaoli, "Evaluation of multi-slice spiral ct scan and image reconstruction technology in estimating costal cartilage volume," Chinese Journal of Reparative and Reconstructive Surgery, vol. 28, no. 10, pp. 1266-1269, 2014.

[4] W. Du, J. Tan, and M. Cai, "Peri operative nursing care of congenital microauricular malformation with autogenous costal cartilage grafting," Journal of Nursing, vol. 25, no. 4, pp. 39-40, 2010.

[5] Y. Ou, B. Li, K. Li, D. Peidong, and Z. Tianyu, "Application of digital technology in reconstruction of auricle in congenital microauricular malformation," Chinese Journal of Medical Aesthetics and Cosmetology, vol. 24, no. 3, pp. 149-152, 2018.

[6] R. L. Flores, H. Liss, S. Raffaelli et al., "The technique for 3D printing patient-specific models for auricular reconstruction," Journal of Cranio-Maxillofacial Surgery, vol. 45, no. 6, pp. 937-943, 2017.

[7] J. Zhou, B. Pan, Q. Yang et al., "Three-dimensional autologous cartilage framework fabrication assisted by new additive manufactured ear-shaped templates for microtia reconstruction," Journal of Plastic, Reconstructive \& Aesthetic Surgery, vol. 69, no. 10, pp. 1436-1444, 2016. 